\title{
Transfer of a matching and nonmatching concept in rats
}

\author{
ESHO NAKAGAWA \\ Kagawa University, Saiwai-Cho, Takamatsu, Kagawa, Japan
}

\begin{abstract}
Transfer of a matching or nonmatching concept across orthogonal dimensions was examined by using stimuli that recurred over trials within each session. Rats were trained a matching or nonmatching task to criterion, and then either reversed on novel stimuli (Group Shifts: matching-nonmatching and nonmatching-matching) or continued with training on a matching and/or nonmatching task with novel stimuli (Group Nonshifts: matching-matching and nonmatching-nonmatching). Performance on the first trial in the transfer between Groups Nonshift (75\% correct) and Shift ( $37.5 \%$ correct) was approximately symmetrically displaced from the chance level. This result suggests that rats transfer a matching or nonmatching concept across pairs of stimuli.
\end{abstract}

Zentall and Hogan (1974) trained some pigeons on matching and others on oddity with one of pair of stimuli, and then transferred them to a new pair of stimuli, either with the same rule as in the first problem (i.e., matching if the first problem was matching or oddity if the first problem was oddity) or with the rule reversed. Pigeons that had not shifted to the opposite task performed better than those that had. That is, nonshifted pigeons learned the shift task more rapidly than did the shifted pigeons. This effect was replicated with a similar shifted versus nonshifted design for a go/no-go version of the matching and oddity task (Zentall \& Hogan, 1975) and in a conventional matching-to-sample experiment using transfer to colors after training on geometric patterns (Zentall \& Hogan, 1976). Zentall and Hogan interpreted these results as demonstrating that pigeons can learn a relational concept. Further evidence appearing to support this claim has been presented by Lombardi, Fachinelli, and Delius (1984), Urcuioli and Nevin (1975), and Urcuioli (1977).

It has also been reported that rats have the ability to form a relational rule. Although Lashley (1938a, 1938b) reported that rats cannot learn an oddity problem, Wodinsky and Bitterman (1953) suggested that rats have such an ability. Nakagawa $(1989,1990)$ has suggested that rats can learn a relational rule in matching and nonmatching (oddity) problems.

Aggleton (1985), Mumby, Pinel, and Wood (1990), and Rothblat and Hayes (1987) showed that rats can generalize matching and nonmatching rules across trial-unique stimuli. When trial-unique (nonrecurring) stimuli are used, rats likely solve the nonmatching-to-sample task by using stimulus-recognition memory (i.e., on the basis of whether the stimulus is familiar or not). Nevertheless,

Requests for reprints should be sent to E. Nakagawa, Department of Psychology, Kagawa University, 1-1 Saiwai-Cho, Takamatsu, Kagawa, 760, Japan. transfer of a matching or nonmatching concept across orthogonal dimensions in tasks with stimuli that recur over trials within each session has not yet been reported in rats. In the case of nonrecurring stimulus items, rats have been required to assess familiarity judgments. By contrast, in the case of recurring stimulus items, rats have been required to assess recency judgments. How the use of recurring stimulus items changes the task from one that assesses familiarity judgments in the case of nonrecurring stimulus items to one that assesses recency judgments in the case of recurring stimulus items has not yet been examined in rats. The present study was designed to investigate transfer of a matching or nonmatching concept across orthogonal dimensions by using stimuli that recurred over trials within each session.

To demonstrate the ability of rats to form an abstract concept that transfers to an orthogonal dimension, a Zentall and Hogan (1974) design was used. By pitting a positive transfer group (nonshifted) against a negative transfer group (shifted), one may be able to detect small transfer effects more easily. If rats learn a matching or nonmatching concept on the basis of a relationship between sample and comparison stimuli and transfer these rules across stimuli, the rats would then choose between a novel pair of stimuli by using the rule learned during initial learning, performing above chance if the rule remains unchanged, and below chance if it changes. Thus, performance on the first trial in the transfer test between Group Nonshift and Group Shift would be symmetrically displaced from the chance level of performance, $50 \%$. In contrast, Aggleton (1985) suggested that it was difficult for rats to acquire and transfer delayed matching or nonmatching when a very restricted set of repeated test stimuli was used. In accordance with Aggleton's suggestion, in the present experiment it should be difficult for rats to acquire a transfer of a matching or nonmatching concept across orthogonal dimensions, because of the use of a very restricted set of repeated test stimuli. 


\section{METHOD}

\section{Subjects}

Thirty-two experimentally naive male Sprague-Dawley rats were used. They were about $\mathbf{2 4 0}$ days old, with an initial average body weight of $423 \mathrm{~g}$. The animals were handled for $5 \mathrm{~min}$ a day for 12 days, and they were maintained on a daily 2 -h feeding schedule each day prior to the experiment. Their daily ration of food was gradually reduced until the body weight of each animal reached $80 \%$ of the baseline weight at the start of the experiment. Water was always available to the animals in their individual home cages. The animals were maintained on a 12:12-h light:dark cycle, with light offset at 4:30 a.m.

\section{Apparatus}

A three-stimulus-presentation T-type jumping stand (shown in Figure 1) was used. The apparatus was painted medium-gray inside and was lit throughout the experiment by a 10 -W fluorescent lamp suspended horizontally $40 \mathrm{~cm}$ above the top of its center. The apparatus consisted of a runway $(25 \times 12 \times 15 \mathrm{~cm}$, length $\times$ width $\times$ height) with a startbox $(20 \times 12 \times 15 \mathrm{~cm})$, two goalboxes $(25 \times 20 \times 15 \mathrm{~cm})$, a center box $(25 \times 16 \times 15 \mathrm{~cm})$, and a jumping stand or platform $(12 \times 54 \mathrm{~cm})$. A piece of cardboard was placed at the entrance of each goalbox and the center box; each piece was $12 \mathrm{~cm}$ square and $15 \mathrm{~cm}$ from the floor, and the pieces were $5 \mathrm{~cm}$ apart edge to edge. A gap over which the animals had to jump $(15 \times 56 \times 15 \mathrm{~cm})$ was located $20 \mathrm{~cm}$ in front of each goalbox. If the rats chose an incorrect stimulus card, they dropped into this gap or stayed on the terrace $(5 \times 12 \mathrm{~cm})$, which was located $5 \mathrm{~cm}$ from the front of the goalbox.
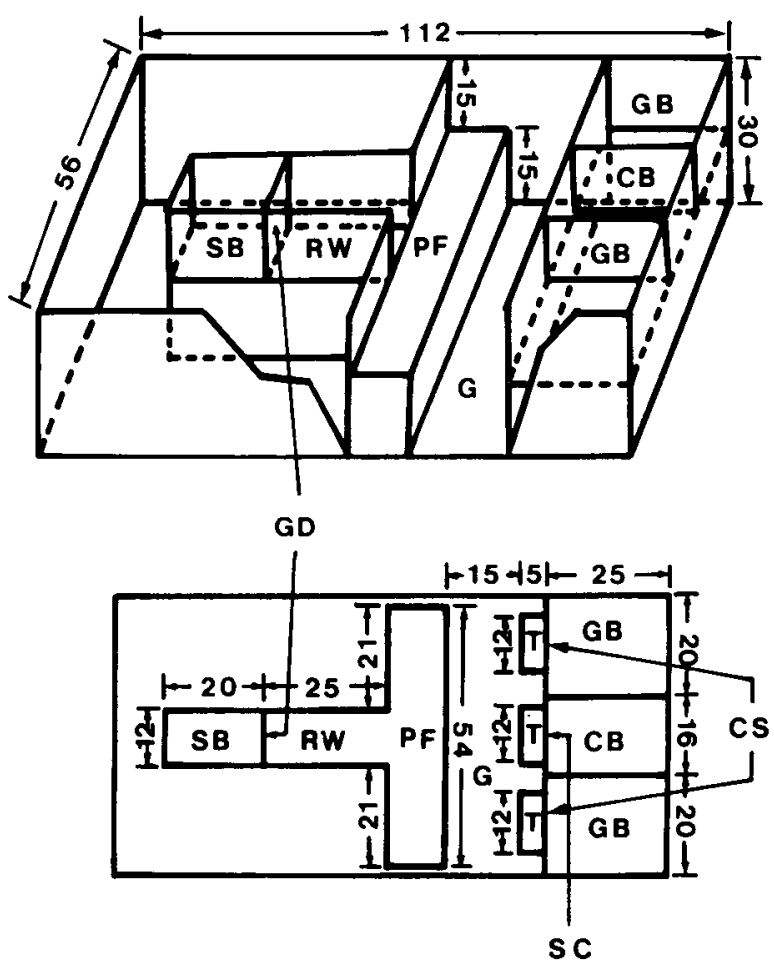

Figure 1. The three-stimulus-presentation T-type jumping stand used in the present experiment. CB, center box; CS, comparison stimulus; G, gap; GB, goalbox; GD, guillotine door; PF, platform; RW, runway; SB, startbox; SC, sample stimulus; T, terrace. (Units $=$ centimeters.)
Stimuli

The stimulus cards were $12-\mathrm{cm}$ squares of cardboard. The sample stimulus card was presented at the entrance of the center box. The comparison stimulus card was presented at the entrance of each goalbox and served as an entrance door. The comparison stimulus cards were placed so that the card serving as the correct door could be pushed down easily, thus permitting the animals to gain entrance into the goalbox, while the card denoting the incorrect door could not. Four stimulus cards were used: the vertically or horizontally striped stimulus cards had alternating black and white lines $1 \mathrm{~cm}$ in width; the white or black stimulus cards were white or black $12-\mathrm{cm}$ squares of cardboard.

\section{Procedure}

The animals were pretrained for 8 days prior to the beginning of the matching or nonmatching learning. On Day 1 , they were allowed to explore the apparatus for two periods of 7 and $5 \mathrm{~min}$. On Days 2-4, they were trained to push down a stimulus card and enter the goalbox to obtain food for 10 daily trials. The gap was not present in this stage of the experiment. On Days 5-8, they were trained to jump over the gap for 10 trials a day. On the last day, all animals jumped over the $15-\mathrm{cm}$ gap. They were given the same number of trials on each goal during this pretraining. Medium-gray stimulus cards were used during this period.

Phase 1 training. The rats received 12 trials/day during Phase 1. Half of the animals were trained on a matching problem to reach a criterion of $11 / 12$ count (Group Matching). The remaining animals were trained on a nonmatching (oddity) problem to reach the criterion (Group Nonmatching). The animals in Group Matching were required to choose a comparison that was the same as a sample stimulus. By contrast, the animals in Group Nonmatching were required to choose a comparison that was different from a sample stimulus. Half of the animals in each group were trained on the white-black stimulus set; the remaining animals were trained on the vertical-horizontal striped stimulus set. In the white-black stimulus set, the white card was the sample stimulus on some trials and the black card was the sample stimulus on the other trials, in random order within each session. In the vertical-horizontal striped stimulus set, the vertically striped card was the sample stimulus on some trials and the horizontally striped card was the sample stimulus on the other trials, in random order within each session. A self-correction method was used, in which, if animals made an error, they were allowed to return to the platform and select the correct stimulus. The position of a positive stimulus followed four predetermined random sequences. The animals were given two $45-\mathrm{mg}$ milk pellets when they made a correct response. The intertrial interval ranged from 4 to $8 \mathrm{~min}$.

Phase 2 transfer. After completing the Phase 1 training, half of the animals in Group Matching were trained on a matching problem with a new stimulus set (Group Nonshift: matching-matching); the remaining half were trained on a nonmatching problem with a new stimulus set (Group Shift: matching-nonmatching). The animals in Group Nonmatching were likewise divided into the two subgroups of Nonshift (nonmatching-nonmatching) and Shift (nonmatching-matching). There was a complete counterbalancing of stimuli within these four groups. Four animals of each group were trained on a vertical-horizontal striped stimulus set, and four were trained on a white-black stimulus set. Other aspects of the procedure were the same as in the Phase 1 training.

\section{RESULTS}

Acquisition of the Phase 1 training by Group Matching was compared with acquisition of the corresponding training in Group Nonmatching. These data are summa- 
rized in Table 1. A $2 \times 2$ analysis of variance (ANOVA) was performed on the days to criterion. The analysis revealed a significant between-group difference $[F(1,28)=$ $22.96, p<.001]$. That is, rats acquired the matching task more rapidly than the nonmatching one.

The means and standard deviations of the number of days to criterion were $16.08(4.87)$ for the white-black stimulus set and $15.46(5.34)$ for the vertical-horizontal striped stimulus set. There was no significant difference in the number of days to criterion in the two stimulus sets $(t<1)$.

Acquisition of the Phase 2 transfer learning by Group Nonshift was compared with acquisition of the corresponding transfer learning in Group Shift. These data are summarized in Figure 2. An ANOVA of group (shift vs. nonshift) and task (matching vs. nonmatching) was performed on the days to criterion. The analysis revealed a significant interaction between group and task $[F(1,28)=15.27, p<.001]$.

Group Nonshift learned the matching task more rapidly than did Group Shift $[F(1,28)=4.97, p<.05]$. By contrast, Group Shift learned the nonmatching task more rapidly than did Group Nonshift $[F(1,28)=10.90$, $p<.003$ ]. Group Nonshift acquired the matching task more rapidly than the nonmatching one $[F(1,28)=4.61$, $p<.05]$. On the other hand, Group Shift acquired the nonmatching task more rapidly than the matching one $[F(1,28)=11.45, p<.002]$.

To examine transfer of rule learning in both the matching task and the nonmatching task, performance on the first trial in the transfer learning was analyzed. Twelve rats of Group Nonshift made a correct response on the first trial in the transfer learning ( $75 \%$ correct). By contrast, 6 rats of Group Shift made a correct response (37.5\% correct). These scores of the two groups were, approximately, symmetrically displaced from the chance level of performance, $50 \%$. A chi-square test was run to analyze differences in performance on the first trial between Nonshift and Shift. The analysis revealed a significant between-group difference $\left[\chi^{2}(1)=4.57, p<.05\right]$.

\section{DISCUSSION}

There is a feature of the task used in the present experiment that might make the findings of transfer across stimuli more significant. When trial-unique (nonrecurring) stimuli are used, rats likely solve the nonmatching-tosample task by using stimulus recognition memory (i.e., on the basis of whether the stimulus is familiar or not). By contrast, the use of stimulus items that recur over trials

Table 1

Means and Standard Deviations of the Number of Days to Criterion in Phase 1 Training

\begin{tabular}{lcl}
\hline \multicolumn{1}{c}{ Group } & $M$ & $S D$ \\
\hline Nonshift (matching-matching) & 16.1 & 4.2 \\
Shift (matching-nonmatching) & 16.0 & 6.6 \\
Nonshift (nonmatching-nonmatching) & 28.9 & 6.2 \\
Shift (nonmatching-matching) & 25.4 & 7.1 \\
\hline
\end{tabular}

10

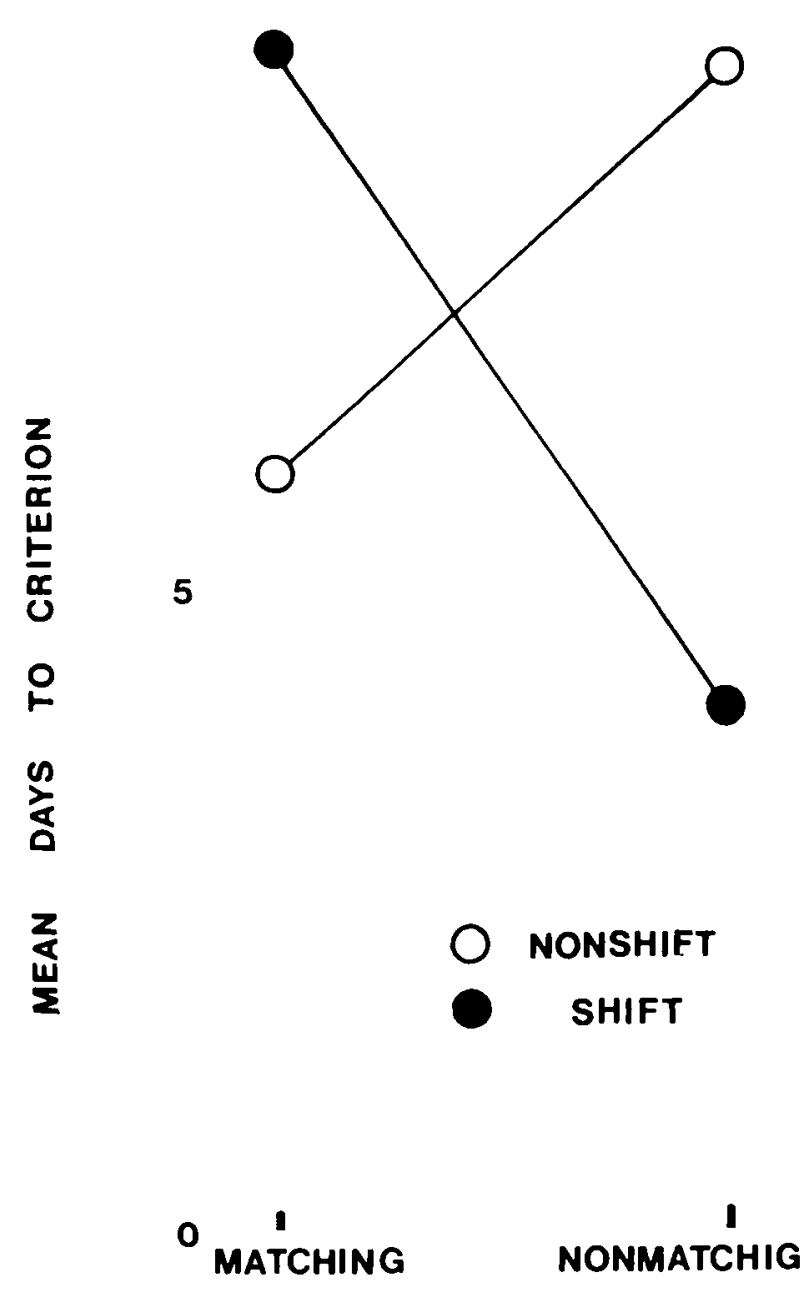

\section{SHIFT TASK}

Figure 2. Mean days to criterion in Phase 2 transfer learning as a function of tasks.

within each session made the tasks require recency discriminations. Transfer of a matching or nonmatching concept across pairs of stimuli in a recency-memory task has not previously been reported. When recurring stimulus items are used, rats likely solve the matching-to-sample and the nonmatching-to-sample task by using recency discriminations (vs. stimulus recognition); this is the novel contribution of the present study.

The first trial data of the transfer test make it clear that rats choose between a novel pair of stimuli by using the rule learned in the Phase 1 training, performing above chance if the rule is unchanged, below chance if it is changed. That is, performance on the first trial between Group Nonshift and Group Shift was, approximately, symmetrically displaced from chance $(50 \%)$ performance. 
The days-to-criterion measure of transfer data (Figure 2) tends to agree with the first data. That is, Group Nonshift learned the matching task more rapidly than did Group Shift. These results replicate the results of Zentall and Hogan $(1974,1975,1976)$. In contrast with Aggleton's (1985) suggestion, rats can solve matching-to-sample and nonmatching-to-sample tasks by using recency discriminations when recurring stimulus items (i.e., a very restricted set of repeated test stimuli) are presented. The present results suggest that rats have the ability to form a matching or nonmatching concept for recurring stimulus items that require rats to assess recency judgments, and that rats transfer the rules acquired in the Phase 1 training to an orthogonal dimension.

The results in the present experiment are consistent with findings from Aggleton (1985), Mumby et al. (1990), and Rothblat and Hayes (1987), who used trial-unique (nonrecurring) stimuli. Thus, the results in the present experiment make it clear that rats can transfer a matching or nonmatching concept to an orthogonal dimension by using stimuli that recur over trials within each session (that is, by using a restricted set of repeated test stimuli) as well as by using trial-unique stimuli.

The results of the training phase (Phase 1) are consistent with findings for young children (Nakagawa, 1988) and for rats (Nakagawa, 1989, 1990). Nevertheless, the results of the training phase in the present experiment are not consistent with findings of Aggleton (1985), Mumby et al. (1990), and Rothblat and Hayes (1987), who all reported a propensity for rats to select the nonmatching stimulus. This discrepancy might reflect differences in the tasks used.

For the nonrecurring stimuli in Aggleton (1985), Mumby et al. (1990), and Rothblat and Hayes (1987), the stimulus value was seldom reversed. On the other hand, in the present experiment, a three-stimulus array (AAB or BBA) was used in which the stimulus value was reversed from trial to trial at random. With the recurring stimuli in the present experiment, rats might acquire a relational rule on the basis of perceptual attributes of stimulus configuration (AAB or BBA). On each trial, rats scan stimulus configurations from left to right or right to left (not necessarily in the same direction from trial to trial). They may classify an adjacent door of the same figure to form a cluster or areas, and may respond to the smaller or larger of the first two areas observed. Thus, the results of the training phase showed a propensity for rats to select the larger of the first two areas observed. By contrast, with nonrecurring stimuli there is no possibility that rats cannot classify an adjacent door of the same figure to form a cluster or areas.

The (non)matching-to-sample paradigms used in Aggleton (1985), Mumby et al. (1990), and Rothblat and Hayes (1987) could all be solved by using tactile as well as visual cues of the samples. By contrast, the apparatus used in the present experiment ensures that rats use visual cues only. As a result, the results in the present study suggest that rats respond correctly on the basis of the visual properties only.
The main finding, however, was that the difference between shifted and nonshifted rats depended on whether they were tested on the matching task or the nonmatching one. That is, rats transferred from matching to nonmatching learned more rapidly than those shifted from nonmatching to nonmatching. By contrast, rats transferred from matching to matching learned more rapidly than those shifted from nonmatching to matching. This asymmetry of transfer effect agrees with the results of experiments done with pigeons (Wilson, Mackintosh, \& Boakes, 1985; Zentall \& Hogan, 1974, 1975). The existence of this asymmetry of transfer effect throws some doubt, however, on Zentall and Hogan's $(1974,1975,1976)$ interpretation of their results. The cause of this asymmetry is unclear, but it seems likely to be related to the inherent bias toward the old stimulus that occurs both in the Phase 1 training and the Phase 2 transfer, as suggested by Wilson et al. (1985).

\section{REFERENCES}

Aggleton, J. P. (1985). One-trial object recognition by rats. Quarterly Journal of Experimental Psychology, 37B, 279-294.

LASHLEY, K. S. (1938a). Conditional reactions in the rat. Journal of Psychology, 6, 311-324.

LASHLEY, K. S. (1938b). The mechanism of vision: XV. Preliminary studies of the rat's capacity for detail vision. Journal of Genetic Psychology, 18, 176-177.

Lombardi, C. M., FAChinelu, C. C., \& DeuUs, J. D. (1984). Oddity of visual patterns conceptualized by pigeons. Animal Learning \& Behavior, 12, 2-6.

Mumby, D. G., Pinel, J. P. J., \& Wood, E. R. (1990). Nonrecurringitems delayed nonmatching-to-sample in rats: $A$ new paradigm for testing nonspatial working memory. Psychobiology, 18, 321-326.

NAKAGAWA, E. (1988). [Shift learning on identity and oddity learning in young children-Examination of validity of House's chain model and Bowers's rule model]. Japanese Journal of Educational Psychology, 36, 333-338.

NAKAGAWA, E. (1989). Matching and oddity learning in white rats. Memoirs of the Faculty of Education, Kagawa University: II, 39, 29-38.

Nakagawa, E. (1990). Transfer of rule learning in rats. Memoirs of the Faculty of Education, Kagawa University: I, 80, 89-104.

Rothblat, L. A., HAYES, L. L. (1987). Short-term object recognition memory in the rat: Nonmatching with trial-unique junk stimuli. Behavioral Neuroscience, 101, 587-590.

UrCuiou, P. J. (1977). Transfer of oddity-from-sample performance in pigeons. Joumal of the Experimental Analysis of Behavior, 27, 195-202.

UrCuioli, P. J., \& Nevin, J. A. (1975). Transfer of hue matching in pigeons. Joumal of the Experimental Analysis of Behavior, 24, 149-155.

Wilson, B., Mackintosh, N. J., Bonkes, R. A. (1985). Matching and oddity learning in the pigeon: Transfer effects and the absence of relational learning. Quarterly Journal of Experimental Psychology, 37B, 295-311.

Wodinsky, J., Bitterman, M. E. (1953). The solution of oddityproblems by the rat. American Joumal of Psychology, 66, 137-140.

Zentall, T. R., \&ogan, D. E. (1974). Abstract concept learning in the pigeon. Journal of Experimental Psychology, 102, 393-398.

Zentall, T. R., HogAN, D. E. (1975). Concept learning in the pigeon: Transfer to new matching and nonmatching stimuli. American Journal of Psychology, 88, 233-244.

Zentall, T. R., \& Hogan, D. E. (1976). Pigeons can learn identity or difference. Science, 191, 408-409.

(Manuscript received February 6, 1992; revision accepted for publication July 8,1992 .) 